\title{
A Retrospective Study of Semen Quality in Young Infertile Men with Androgenetic Alopecia after Discontinuation of Finasteride
}

\author{
Yumi Inoue ${ }^{1}$, Makoto Yono ${ }^{1,2}$, Megumi Kimura ${ }^{1}$, Masaharu Hori ${ }^{1}$, Shigeki Tsuji ${ }^{1}$, Takanori Tanaka ${ }^{1}$, \\ Yukikuni Sakata $^{1}$, Shin Irie ${ }^{1}$
}

${ }^{I}$ Department of Clinical Pharmacology, Nishi-Kumamoto Hospital, SOUSEIKAI, Japan, ${ }^{2}$ Department of Urology, NishiKumamoto Hospital, SOUSEIKAI, Japan

Objectives: Androgenetic alopecia (AGA), male pattern hair loss, is caused by androgen-dependent miniaturization of scalp hair follicles. Finasteride, an inhibitor of type II $5 \alpha$-reductase, decreases the conversion of testosterone to dihydrotestosterone, slows progressive hair loss and increases hair growth in men with AGA. Because androgens are essential for fertility and finasteride is associated with mild decreases in semen parameters that appear reversible after discontinuation, we evaluated semen quality in young male patients with finasteride-associated infertility to determine whether all semen parameters are reversible after discontinuation.

Methods: In a retrospective study, the records of 20 young male patients with finasteride-associated infertility between April 2007 and March 2017 were evaluated. Serum follicle-stimulating hormone, luteinizing hormone and testosterone levels, prostate volume, and semen parameters (semen volume, sperm concentration, total sperm number and total motility) were assessed before and after discontinuation of finasteride.

Results: At initial evaluation, young infertile men treated with finasteride had relatively high testosterone levels and low semen quality. After discontinuation of finasteride, sperm concentration and total motility increased to or toward the normal range, whereas semen volume did not change over a 24 -week period. Although finasteride has been shown to reduce the volume of the prostate even in young men without benign prostatic hyperplasia, finasteride discontinuation did not increase prostate volume.

Conclusions: The effects of finasteride on semen volume and prostate volume in young infertile men were not reversible over the 24-week off-drug period. Androgens are necessary for maintaining the structure and secretory function of adult male sex organs such as the seminal vesicle and prostate. Androgen deprivation has been shown to reduce the volume of the seminal vesicle and prostate. These findings suggest that finasteride may reduce the volume of the seminal vesicle as well as prostate resulting in decreased semen volume. This atrophy may be of importance to explain semen volume after discontinuation of finasteride. Dutasteride, an inhibitor of both type I and type II $5 \alpha$-reductase, has recently been approved in Japan for the treatment of AGA. Therefore, clinical judgment should be exercised when these drugs are given to young men who desire fertility. 